\title{
Video-Assisted Thoracic Surgery for Esophagus for Treating Locally Recurrent Gastric Cancer at the Anastomotic Site
}

\author{
Hitoshi Ojima ${ }^{a}$ Akihiko Sano $^{\text {a }}$ Yasuyuki Fukai ${ }^{a}$ Makoto Soda ${ }^{a}$ \\ Hiroyuki Kuwano ${ }^{\text {b }}$ \\ ${ }^{a}$ Department of Gastroenterological Surgery, Gunma Prefectural Cancer Center, Ota, and \\ ${ }^{b}$ Department of General Surgical Science, Gunma University Faculty of Medicine, Maebashi, Japan
}

\section{Key Words}

Recurrent gastric cancer - Total gastrectomy .

Video-assisted thoracic surgery for esophagus

\section{Abstract}

Objective: To report a case of video-assisted thoracic surgery for esophagus (VATS-E) for the treatment of locally recurrent gastric cancer at the anastomotic site. Clinical Presentation and Intervention: A 59-year-old man underwent total gastrectomy for gastric cancer. Histopathologically, it was mucinous adenocarcinoma [pT1(sm), pN1, sM0, PM(-), $\mathrm{DM}(-)$, stage IB]. Local recurrence was detected and he received chemotherapy and radiotherapy; histopathologically, it was identified as mucinous adenocarcinoma [pT1(sm), pNO, sM0, stage IA]. As the local recurrence was uncontrollable, he underwent VATS-E. At the 1-year follow-up, he was recurrence-free. Conclusion: The VATS-E minimally invasive surgery was a very useful technique for anastomotic recurrence after total gastrectomy in our patient.

\section{KARGER}

E-Mail karger@karger.com www.karger.com/mpp

\section{() 2013 S. Karger AG, Basel} 1011-7571/13/0232-0180\$38.00/0

Karger

Open access

This is an Open Access article licensed under the terms of the Creative Commons Attribution-NonCommercial 3.0 Unported license (CC BY-NC) (www.karger.com/OA-license), applicable to the online version of the article only. Distribution permitted for non-commercial purposes only.

\section{Introduction}

Gastric cancer remains the most common cause of cancer deaths in Japan. Curative treatment of gastric cancer requires the complete excision of the tumor and lymph node dissection. Despite radical surgery and the development of adjuvant chemotherapy, recurrence is common in patients undergoing curative resection [1]. The high incidence of recurrence may be attributed to the clinicopathological characteristics of the tumor $[2,3]$. Curative surgery for recurrent gastric cancer is possible in only a very few cases of anastomotic recurrence identified in the early stage [4]. Other cases are usually administered chemotherapy and/or radiotherapy and many are not cured completely [5]. We report the case of a patient who underwent video-assisted thoracic surgery for esophagus (VATS-E) for the treatment of locally recurrent gastric cancer at the anastomotic site.
Dr. Hitoshi Ojima

Department of Gastroenterological Surgery Gunma Prefectural Cancer Center 617-1 Takabayashi-Nishi, Ota, Gunma 373-8550 (Japan) E-Mail hiojima@gunma-cc.jp 

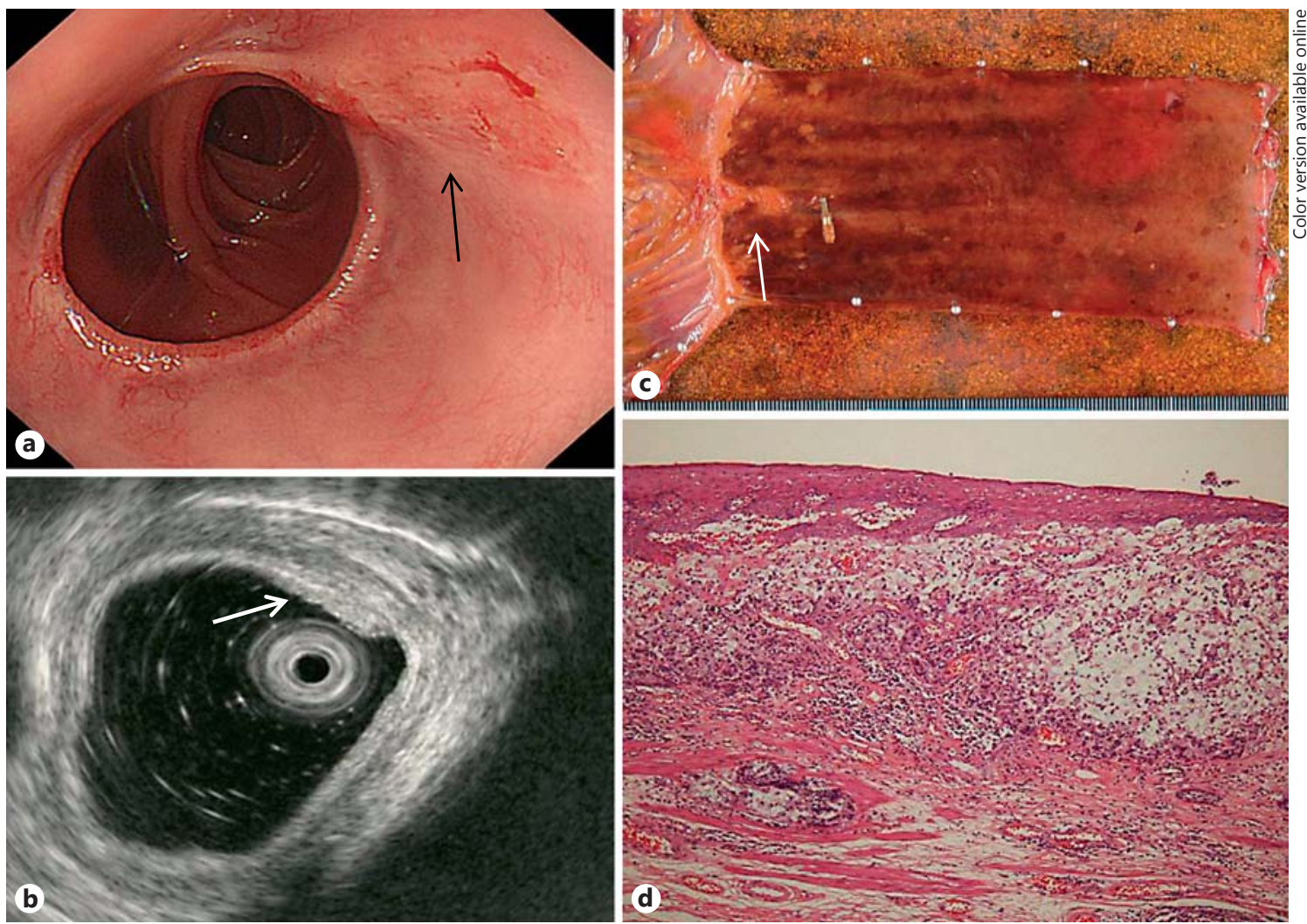

Fig. 1. a Endoscopic image showing a slightly depressed-type tumor at the esophageal side of the esophagojejunal anastomosis. b Endoscopic ultrasonography showing thickening of the submucosal layer. c The surgically resected specimen revealing a slightly depressed-type tumor $(18 \times 15 \mathrm{~mm})$. The tumor extending from the anastomotic region to the esophageal side. $\mathbf{d}$ Histopathological findings of the resected specimen revealing signet-ring cell carcinoma invading the submucosal layer.

\section{Case Report}

The patient was a 59-year-old man with a history of total gastrectomy due to Roux-en-Y reconstruction for gastric cancer (U, Less, 0 -IIc) 1 year before. The histological diagnosis was mucinous adenocarcinoma ( $\mathrm{pT} 1, \mathrm{pN} 1, \mathrm{sH} 0, \mathrm{sP} 0, \mathrm{sM} 0$, ly2, v1, stage IB). Both the proximal and distal margins were negative for cancer. Anastomotic leakage was observed postoperatively, resulting in left thoracic empyema, and the patient underwent a drainage operation. One year after the resection, recurrent tumor was observed at the esophageal side of the esophagojejunal anastomosis. The patient did not want a surgical resection, so he underwent chemotherapy with the oral anticancer agent S-1. Although the local recurrence became a transient complete response, the tumor recurred 2 years later. Radiotherapy was administered at a dose of $60.5 \mathrm{~Gy}$, followed by administration of the oral anticancer drug UFT; however, the local recurrence was uncontrollable and the patient was referred to our hospital.

Computed tomography revealed a slight thickening at the anastomotic area, and no distant metastases were observed. Endoscopy showed a slightly depressed-type tumor at the esophageal side of esophagojejunal anastomosis (fig. 1a), with a diameter of about 20 $\mathrm{mm}$. Endoscopic ultrasonography showed thickening of the submucosal layer (fig. 1b). Because of the left thoracic empyema, left tho- racotomy was expected to be more difficult, so we performed VATSE. There were slight adhesions at the lower mediastinum of an anastomotic region, but exfoliation was comparatively easy. Although we dissected regional lymph nodes, lymph nodes metastases were not detected at the thoracic and abdominal cavity. Reconstruction after esophagectomy was performed by colon interposition using the right hemicolon by the subcutaneous route, along with vascular anastomoses. The total operative time was $512 \mathrm{~min}$ and blood loss was $630 \mathrm{ml}$. Macroscopically, the tumor was a slightly depressedtype tumor, measuring $18 \times 15 \mathrm{~mm}$, extending to the esophagus from the anastomosis (fig. 1c). On the basis of histopathological examination, mucinous adenocarcinoma ( $\mathrm{pT} 1, \mathrm{pN} 0, \mathrm{sH} 0, \mathrm{sP} 0, \mathrm{sM} 0$, PM-, DM-, stage IA) was diagnosed (fig. 1d). The patient was discharged 19 days after the operation after an uneventful course and was recurrence-free at a 1-year follow-up.

\section{Discussion}

Recurrence after the curative gastrectomy of this patient was similar to such recurrences reported previously $[1,2,5,6]$. A probable explanation for the local recurrence 
of the tumor in this patient could be due to lymphatic vessel invasion which was detected in the gastric wall and because mucinous adenocarcinoma has a strong potential for metastasis and recurrence [7]. The surgical procedures necessary for gastric cancer meant that implantation also seemed likely to have been the mechanism of recurrence. It is important to avoid contamination when using automatic stapling instruments and anastomosis devices.

Re-excision, chemotherapy and chemoradiation therapy are all possibilities for treating local recurrence. However, there are very few cases appropriate for re-excision because the postoperative mortality and morbidity are unacceptable [4]. There have been reports that early detection of asymptomatic gastric cancer recurrence did not improve the overall survival of patients with recurrence after curative resection [8]. However, early detection of local recurrence is required for performing re-excision so that curative resection may be attained. Routine follow- up to detect asymptomatic recurrence is important after surgery for gastric cancer. Although re-excision would have been considered as the next step, left thoracotomy was anticipated to be very difficult because of the history of empyema following the prior operation. Since complete excision was considered, we did not choose re-resection by a transhiatal approach in this case. VATS-E is minimally invasive surgery compared to thoracotomy, so we chose VATS-E of the left lateral position which meant we did not have any difficulties related to the left thoracic empyema.

\section{Conclusion}

The VATS-E minimally invasive surgery was a useful technique for anastomotic recurrence after total gastrectomy in our patient. It is important to detect the local recurrence as early as possible.

\section{References}

1 D’Angelica M, Gonen M, Brennan MF, et al: Patterns of initial recurrence in completely resected gastric adenocarcinoma. Ann Surg 2004;240:808-816.

$\checkmark 2$ Wu CW, Lo SS, Shen KH, et al: Incidence and factors associated with recurrence patterns after intended curative surgery for gastric cancer. World J Surg 2003;27:153-158.

3 Moriguchi S, Maehara Y, Korenaga D, et al: Risk factors which predict pattern of recurrence after curative surgery for patients with advanced gastric cancer. Surg Oncol 1992;1: 341-346.
-4 Carboni F, Lepiane P, Santoro R, et al: Treatment for isolated loco-regional recurrence of gastric adenocarcinoma: does surgery play a role? World J Gastroenterol 2005;11:70147017.

5 Lim DH, Kim DY, Kang MK, et al: Patterns of failure in gastric carcinoma after D2 gastrectomy and chemoradiotherapy: a radiation oncologist's view. Br J Cancer 2004;91:11-17.
-6 Otsuji E, Kuriu Y, Ichikawa D, et al. Time to death and pattern of death in recurrence following curative resection of gastric carcinoma: analysis based on depth of invasion. World J Surg 2004;28:866-869.

7 Nishimura M, Honda I, Watanabe S, Nagata $M$, et al: Recurrence in jejunal pouch after proximal gastrectomy for early upper gastric cancer. Gastric Cancer 2003;6:197-201.

$>8$ Kodera Y, Ito S, Yamamura Y, et al: Follow-up surveillance for recurrence after curative gastric cancer surgery lacks survival benefit. Ann Surg Oncol 2003;10:898-902. 